Pacific Journal of Mathematics

DISSIPATIVE OPERATORS IN A BANACH SPACE 


\title{
DISSIPATIVE OPERATORS IN A BANACH SPACE
}

\author{
G. Lumer and R. S. Phillips
}

1. Introduction. The Hilbert space theory of dissipative operators ${ }^{1}$ was motivated by the Cauchy problem for systems of hyperbolic partial differential equations (see [5]), where a consideration of the energy of, say, an electromagnetic field leads to an $L_{2}$ measure as the natural norm for the wave equation. However there are many interesting initial value problems in the theory of partial differential equations whose natural setting is not a Hilbert space, but rather a Banach space. Thus for the heat equation the natural measure is the supremum of the temperature whereas in the case of the diffusion equation the natural measure is the total mass given by an $L_{1}$ norm. In the present paper a suitable extension of the theory of dissipative operators to arbitrary Banach spaces is initiated.

An operator $A$ with domain $\mathfrak{D}(A)$ contained in a Hilbert space $H$ is called dissipative if

$$
r e(A x, x) \leqq 0, \quad x \in \mathfrak{D}(A),
$$

and maximal dissipative if it is not the proper restriction of any other dissipative operator. As shown in [5] the maximal dissipative operators with dense domains precisely define the class of generators of strongly continuous semi-groups of contraction operators (i.e. bounded operators of norm $\leqq 1$ ). In the case of the wave equation this furnishes us with a description of all solutions to the Cauchy problem for which the energy is nonincreasing in time. Our aim will be to characterize the generators of all strongly continuous semigroups of contraction operators in an arbitrary Banch space. For this we shall use the notion of a semi innerproduct, introduced in [4].

DeFinition 1.1. A semi inner-product is defined on a complex (real) vector space $\mathfrak{X}$ if to each pair $x, y$ in $\mathfrak{X}$ there corresponds a complex (real) number $[x, y]$ in such a way that:

$$
\begin{array}{rr}
{[x+y, z]=[x, z]+[y, z] \quad x, y, z \in \mathfrak{X} \text { and } \lambda \text { complex }(\text { real }) ;} \\
{[\lambda x, y]=\lambda[x, y]} & \text { for } x \neq 0 ; \\
\mid[x, x]>0 & x, y \in \mathfrak{X} .
\end{array}
$$

Received June 7, 1960. The second named author wrote this paper under the sponsorship of the National Science Foundation, contract NSF G 6330.

1 The term operator will be understood throughout as denoting a linear transformation, not necessarily bounded, with domain and range subspaces of the same space. 
Such an $\mathfrak{X}$ is called a semi inner-product space (in short a s.i.p.s.).

One easily shows (see [4]) that any s.i.p.s. is a normed space, the norm being defined by $|x|=[x, x]^{1 / 2}$.

Conversely any Banach (or normed) space can be made into a s.i.p.s.and this in general in infinitely many ways-as follows: Let $\mathfrak{X}$ denote a Banach space with adjoint space $\mathfrak{X}^{*}$. According to the Hahn Banach theorem to each $x \in \mathfrak{X}$ there corresponds at least one (and let us choose exactly one) bounded linear functional $W x \in \mathfrak{X}^{*}$ such that $(x, W x) \equiv$ $W x(x)=|x|^{2}$. Then clearly $[x, y]=(x, W y)$ for each $x, y \in \mathfrak{X}$ defines a semi inner-product on $\mathfrak{X}$. It is clear that there is a unique semi innerproduct compatible with the norm of a given Banach space if and only if its unit sphere is "smooth" in the sense that there is a unique supporting hyperplane at each point. In particular for a Hilbert space the only semi inner-product is the usual inner-product.

Definition 1.2. An operator $A$ with domain $\mathfrak{D}(A)$ in a s.i.p.s. $\mathfrak{X}$ is called dissipative if

$$
r e[A x, x] \leqq 0, \quad x \in \mathfrak{D}(A) .
$$

DeFINITION 1.3. For any operator $A$ with domain $\mathfrak{D}(A)$ in a s.i.p.s. $\mathfrak{X}$, we define its numerical range $W(A)$ as

$$
W(A) \equiv[[A x, x] ; \quad x \in \mathfrak{D}(A), \quad|x|=1],
$$

and set

$$
\theta(A) \equiv \sup r e\{W(A)\} .
$$

In the sequel, although the connection between dissipative operators and semi-group of contraction operators is always kept in the foreground, only $\S \S 3,4$ and 5 deal primarily with semi-group theory. Section 2 is concerned with bounded dissipative operators and their relation to the geometry of the unit sphere $S$ of $\mathfrak{B}(\mathfrak{X})$, the algebra of all bounded operators on $\mathfrak{X}$. The following identity, previously proved in [4], is basic for these considerations:

$$
\theta(A)=\lim _{t \rightarrow 0^{+}} t^{-1}(|I+t A|-1),
$$$$
A \in \mathfrak{B}(X)
$$

here $I$ is the identity operator. ${ }^{2}$ In particular, we show that a conjecture

${ }^{2}$ The relation $(1.7)$ is readily verified when $\mathfrak{X}$ is a Hilbert space. In fact for $x \in \mathfrak{X}$, $|x|=1$, and $t>0$, we have

$$
|(I+t A) x|^{2}=(x+t A x, x+t A x)=1+2 \operatorname{tre}(A x, x)+t^{2}|A x|^{2} .
$$

As a consequence

and also

$$
|I+t A|^{2} \leqq 1+2 t \theta(A)+t^{2}|A|^{2}
$$

Hence

$$
2 r e(A x, x) \leqq t^{-1}\left(|I+t A|^{2}-1\right)+t|A|^{2} .
$$

$$
\theta(A)=\lim _{t \rightarrow 0^{+}}(2 t)^{-1}\left(|I+t \mathrm{~A}|^{2}-1\right)=\lim _{t \rightarrow 0^{+}} t^{-1}(|I+t A|-1) .
$$


of Bohnenblust and Karlin [1]-concerning the non-existence of tangent rays to $S$ at $I$ in the direction of the radical-is false. On the other hand we show that if a suitable condition (on the growth of the resolvent of the quasi-nilpotent operator in question) is assumed, then the conclusion of the conjecture is valid; in particular, the conjecture always holds in the finite dimensional case.

The relation (1.7) is also of interest in semi-group theory. In fact, for a strongly continuous semi-group of operators $[S(t) ; t \geqq 0]$ with infinitesimal generator $A$, if we define the local type $\omega(A)$ as

$$
\omega(A)=\lim _{t \rightarrow 0^{+}} t^{-1} \log |S(t)|
$$

(see [3; Theorem 7.11.1]), then for $A \in \mathfrak{B}(\mathfrak{X})$ and $S(t)=\exp (t A)$, it is clear that

$$
\omega(A)=\lim _{t \rightarrow 0^{+}} t^{-1}(|I+t A|-1),
$$

and hence by (1.7) that

$$
\omega(A)=\theta(A) .
$$

This result extends to the case of unbounded $A$ as does the following generalization of (1.7) (proved in $\S 3$ )

$$
\text { (1.10) } \sup _{\substack{\left.|x|=1 \\ x \in \mathbb{D}^{(} A^{2}\right)}} \lim _{t \rightarrow 0^{+}} t^{-1}(|x+t A x|-1) \leqq \theta(A) \leqq \sup _{\substack{|x|=1 \\ x \in \mathbb{D}^{(\boldsymbol{A})}}} \lim _{t \rightarrow 0^{+}} t^{-1}(|x+t A x|-1) \text {. }
$$

Section 3 deals with unbounded dissipative operators and their connection with the generation of semi-groups of contraction operators. It is shown that an operator $A$ with dense domain in a s.i.p.s. $\mathfrak{X}$ generates a strongly continuous semi-group of contraction operators if and only if it is dissipative and the range of $I-A$, in symbols $\Re(I-A)$, is $\mathfrak{X}$. In particular this will be the case if $A$ is closed, densely defined, and both $A$ and $A^{*}$ are dissipative. It is clear that any dissipative operator has a maximal dissipative extension. Now if $A$ is a dissipative operator with dense domain in a Hilbert space then $\Re(I-A)$ is $\mathfrak{X}$ if and only if $A$ is maximal dissipative. Unfortunately the situation is not that simple in general; we show by example that $A$ can be maximal dissipative with dense domain and yet $\Re(I-A)$ need not be dense in $\mathfrak{X}$. On the other hand, it is shown that $A$ generates a semi-group of contraction operators if it is dissipative and if the set $\left[x ; x \in \mathfrak{D}\left(A^{\infty}\right),\left|A^{n} x\right|^{1 / n}=o(n)\right]$ is dense in $\mathfrak{X}$. It is clear that the above condition holds for self-adjoint operators on a Hilbert space, or more generally that it holds for all unbounded spectral operators in the sense of Dunford. It is shown that this condition also holds for all generators of groups of operators, but not for all generators of semi-groups. Two lemmas concerning the smallest closed 
extension of a dissipative operator with dense domain are given at the end of $\S 3$.

Finally it was noticed that in certain physical initial value problems, a given generator could be, roughly speaking, dissipative with respect to several norms simultaneously. In a cooling process, for instance, the maximum temperature is nonincreasing as is the total amount of heat in the body; thus the semi-group solution consists of contraction operators in both an $L_{\infty}$ and an $L_{1}$ setting. With this as motivation, it is shown in $\S 5$ that if $[S(t)]$ defines a semi-group of operators of finite local type $\omega_{p}$ on $L_{p}$ and of finite local type $\omega_{q}$ on $L_{q}, 1 \leqq p \leqq q \leqq \infty$, then as a semi-group acting on $L_{p} \cap L_{q}$ it can be extended to be a semigroup of local type $\omega_{s} \leqq \alpha \omega_{p}+\beta \omega_{q}$ on $L_{s}$; here $\alpha, \beta \geqq 0, \alpha+\beta=1$, and $s^{-1}=\alpha p^{-1}+\beta q^{-1}$. A similar convexity type result involving conditions on the generators rather than the semi-group operators is also. established.

2. Bounded dissipative operators. This section is concerned with bounded dissipative operators on a Banach space $\mathfrak{X}$. We show first of all that $A \in \mathfrak{B}(\mathfrak{X})$ is dissipative if and only if the semi-group exp $(t A)$. generated by $A$ is a semi-group of contraction operators. Next we consider the special class of dissipative operators for which $\sup r e\{W(A)\}=$ 0 in connection with the geometrical properties of the unit sphere of $\mathfrak{B}(\mathfrak{X})$. Among other things we disprove a conjecture of $\mathrm{H}$. F. Bohnenblust and S. Karlin [1] by exhibiting a non-trivial quasi-nilpotent bounded dissipative operator.

LeMma 2.1. If $A$ is a bounded dissipative operator on a s.i.p.s. $\mathfrak{X}$, then the resolvent of $A$, namely $R(\lambda, A)$ exists for $r e \lambda>0$.

Proof. Denote the spectrum of $A$ by $\sigma(A)$ and suppose $\lambda$ is a point in the boundary of $\sigma(A)$. Then there exists a sequence $\left\{x_{n}\right\} \subset \mathfrak{X},\left|x_{n}\right|=1$, such that $(\lambda I-A) x_{n} \rightarrow 0$. It follows that $\left[\lambda x_{n}-A x_{n}, x_{n}\right]=\lambda-\left[A x_{n}, x_{n}\right] \rightarrow$ 0 , and since $r e\left[A x_{n}, x_{n}\right] \leqq 0$, we see that $r e \lambda \leqq 0$; consequently the spectrum itself if contained in the half plane $r e \lambda \leqq 0$.

Combining Lemma 2.1 and Theorem 3.1 we have

Theorem 2.1. A bounded operator on a s.i.p.s. generates a semigroup of contraction operators if and only if it is dissipative.

REMARK 1. In the previous theorem all that is required is to show that $|\exp (t A)| \leqq 1$ if and only if $A$ is dissipative. This however is an immediate consequence of the relation (1.9) which in turn depends on (1.7) or, equivalently, on (3.8). 
REMARK 2. Theorem 2.1 implies also that if $A$ is a bounded dissipative operator with respect to any s.i.p. consistent with the norm of a Banach space $\mathfrak{X}$, then it is dissipative with respect to any other such s.i.p.. This fact could also be obtained from the corollary of Lemma 3.2 ; it is also a consequence of a general linear space lemma given in the appendix.

In considering the geometry of the unit sphere $S$ of $\mathfrak{B}(\mathfrak{X})$, we first recall some terminology. Let $I$ be the identity operator, and $A \in \mathfrak{B}(\mathfrak{X})$, $A \neq 0$, we refer to $\{I+t A: t \geqq 0\}$ as the "ray generated by $I$ and $A$." According to [1], the ray generated by $I$ and $A$ will be said to be tangent to $S$ at $I$, if

$$
\lim _{t \rightarrow 0^{+}} t^{-1}(|I+t A|-1)=0 .
$$

We have then as an immediate consequence of (1.9) and (3.8).

Lemma 2.2. The ray generated by $I$ and $A$ is tangent to $S$ at $I$, if and only if sup $r e\{W(A)\}=0$ (or equivalently, if and only if $A$ generates a semi-group of local type 0 ).

Inspecting the proof of Lemma 2.1, we see at once that if in particular $A$ is quasi-nilpotent, then $\sup r e\{W(A)\} \geqq 0$. Hence we also have

CoROLLARY. A quasi-nilpotent bounded operator generates with I a ray tangent to $S$ at $I$, if and only if it is dissipative.

Bohnenblust and Karlin conjecture in [1] that in no Banach algebra can there exist a ray tangent to the unit sphere at the identity which is generated by an element of the radical. In order to show that this conjecture is false, it suffices, in view of the above corollary, to exhibit a non-trivial quasi-nilpotent dissipative operator $A$ on a Hilbert space $\mathfrak{X}$, considering $A$ to belong to some commutative subalgebra of $\mathfrak{B}(\mathfrak{X})$ containing $I$ and $A$.

THEOREM 2.2. There exists a bounded quasi-nilpotent dissipative operator $A$ on a Hilbert space $\mathfrak{X}$, different from 0 .

Proof. Let $\mathfrak{X} \equiv L^{2}[0,1]$. And set

$$
g(\sigma, \tau)= \begin{cases}\sigma(1-\tau) & \text { for } 0 \leqq \sigma \leqq \tau \leqq 1 \\ \tau(1-\sigma) & \text { for } 0 \leqq \tau \leqq \sigma \leqq 1\end{cases}
$$

This is the Green's function corresponding to the boundary value problem $-\left(d^{2} / d t^{2}\right), x(0)=x(1)=0$. Thus the operator $G$ defined by

$$
(G x)(\sigma)=\int g(\sigma, \tau) x(\tau) d \tau,
$$


is selfadjoint and positive. Now consider

$$
a(\sigma, \tau)= \begin{cases}0 & \text { for } \sigma \leqq \tau \\ g(\sigma, \tau) & \text { for } \tau \leqq \sigma\end{cases}
$$

and define

$$
(A x)(\sigma)=-\int a(\sigma, \tau) x(\tau) d \tau,
$$

$x \in \mathfrak{X}$.

Then $A+A^{*}=-G$, hence $r e(A x, x) \leqq 0$ for $x \in \mathfrak{X}$, so that $A$ is dissipative. Moreover it is easily verified that $\left|A^{n}\right|^{1 / n} \rightarrow 0$, and the proof is complete.

TheORem 2.3. Let $A$ be a bounded quasi-nilpotent operator on a s.i.p.s. $\mathfrak{X}$, whose numerical range $W(A)=\{[A x, x] ;[x, x]=1\}$ is confined to an angular region $\Omega$, with vertex at 0 and angle $\pi / \alpha, 0 \leqq \pi / \alpha<2 \pi$. If $|R(\lambda, A)|=0\left(\exp |\lambda|^{-\beta}\right), \beta<\alpha$, as $\lambda \rightarrow 0$ in some angular region strictly containing $\Omega$, then $A=0$.

Proof. For the sake of clarity let us use $\Omega_{3}$ to denote the angular region (in the complex plane) with vertex at 0 and angle $\theta$, bisected by the real axis. We may assume without loss of generality that $\Omega=\Omega_{\pi / a}$.

Next we consider $F(\lambda)=(I+\lambda A)^{-1}$. Since $A$ is quasi-nilpotent, $F(\lambda)$ is an entire function in the complex variable $\lambda$. We shall first show that $|F(\lambda)|=O(1)$ as $\lambda \rightarrow 0$ for $\lambda \in \Omega_{\theta}$ with $\theta<2 \pi-\pi / \alpha$. For $x \in \mathfrak{X},[x, x]=1$,

$$
|(I+\lambda A) x| \geqq|[x+\lambda A x, x]|=|1+\lambda[A x, x]|
$$

If $\lambda \in \Omega_{\theta}$, then $1+\lambda[A x, x] \in 1+\Omega_{(\pi / \alpha)+\theta}$, and the minimum distance $M(\theta)$ from the origin to the latter set is positive. It follows that

$$
\begin{array}{ll}
|(I+\lambda A) x| \geqq M(\theta)|x| & \text { for } \quad x \in \mathfrak{X} \\
|F(\lambda)| \leqq \frac{1}{M(\theta)}, & \text { for } \quad \lambda \in \Omega_{\text {子 }} .
\end{array}
$$

On the other hand we have

$$
|F(\lambda)|=\frac{1}{|\lambda|}\left|R\left(-\lambda^{-1}, A\right)\right|=0\left(\exp |\lambda|^{\beta}\right)
$$

as $\lambda \rightarrow \infty$ in a region $-\Omega_{\varphi}, \varphi>\pi / \alpha$. We now choose $\theta<2 \pi-\pi / \alpha$ in such a way that the complement of $\Omega_{\theta}$ (i.e. $-\Omega_{2 \pi-\theta}$ ) is contained strictly in both $-\Omega_{\varphi}$ and $-\Omega_{\pi / \beta}$. Then

$$
\begin{array}{ll}
|F(\lambda)|=O(1) & \text { in } \Omega_{\theta} \\
|F(\lambda)|=0\left(\exp |\lambda|^{\beta}\right) & \text { as } \lambda \rightarrow \infty \text { in }-\Omega_{2 \pi+\theta} .
\end{array}
$$


Under these conditions one can apply the Phragmen-Lindelof theorem to the numerical valued functions $f(F(\lambda)), f$ being any bounded linear functional on the algebra $\mathfrak{B}(\mathfrak{X})$ of all bounded operators on $\mathfrak{X}$. One concludes that $|f(F(\lambda))|=O(1)$ in the entire plane, hence $f(F(\lambda))=$ $f(F(0))=f(I) ; F(\lambda) \equiv I$, and consequently $A=0$.

CoRollary. If $A$ is a dissipative, quasi-nilpotent bounded operator on a s.i.p.s., and $|R(\lambda, A)|=0\left(\exp |\lambda|^{-1+\varepsilon}\right), \varepsilon>0$, as $\lambda \rightarrow 0$ in some angular region strictly containing the half plane $r e \lambda \leqq 0$, then $A=0$.

Notice that for the operator $A$ defined in (2.2) one verifies easily that $|R(\lambda, A)|=0\left(\exp |\lambda|^{-1}\right)$ near 0 ; which shows that the condition in Theorem 2.3 is sharp.

We also have the following.

Corollary. If $A$ is a quasi-nilpotent, dissipative, and $\mathfrak{X}$ is finite dimensional, then $A=0$.

Proof. In this case $R(\lambda, A)$ is a polynomial in $\lambda^{-1}$, hence $|R(\lambda, A)|=$ $0\left(\exp |\lambda|^{-\delta}\right), \delta>0$, near 0 .

Next, using the fact that a dissipative bounded operator generates a semi-group of contractions we obtain another condition under which a dissipative quasi-nilpotent bounded operator vanishes.

THEOREM 2.3. Let $A$ be a bounded quasi-nilpotent dissipative operator defined on a s.i.p.s. $\mathfrak{X}$. If for some $k \geqq 0$

$$
\exp (t A)=O\left(t^{k}\right)
$$

as $t \rightarrow-\infty$, then $A=0$.

Proof. It is known (see [3; Theorem 3.13.8]) that an entire vectorvalued function $F(z)$ of order one and of minimal type such that $|F(t)|=$ $O\left(|t|^{k}\right)$ for real $t$, is necessarily a polynomial in $z$ of degree $<k$. Setting $F(z)=\exp (z A)$ we see that

$$
|F(z)| \leqq \exp (|z||A|)
$$

so that $F(z)$ is of order one. Moreover, since $A$ is quasi-nilpotent, given $\varepsilon>0$ there is an $N_{\varepsilon}$ such that $\left|A^{n}\right| \leqq \varepsilon^{n}$ for all $n>N_{\varepsilon}$. Hence

$$
|F(z)| \leqq \sum_{n=1}^{N_{\varepsilon}}|z|^{n}|A|^{n} \mid n !+e^{\varepsilon|z|} \leqq e^{2 \varepsilon|z|}
$$

for $|z|$ sufficiently large. Therefore

$$
\limsup _{r \rightarrow \infty} r^{-1} \sup [\log |F(z) ;| z \mid=r] \leqq 2 \varepsilon,
$$


and $\varepsilon$ being arbitrary, it follows that $F(z)$ is of minimal type. Now $A$ is dissipative and hence $|F(t)| \leqq 1$ for real $t>0$. By hypothesis $|F(t)|=$ $O\left(|t|^{k}\right)$ for real negative $t$. Thus the above mentioned result applies. and we may conclude that $F(z)$ is a polynomial, say of degree $m$. But then

$$
\liminf _{|z| \rightarrow \infty}\left|z^{-m} F(z)\right|>0 \text {. }
$$

In particular this holds for real positive $z$ so that $m=0$. In other words $\exp (t A) \equiv F(0)=I$ and $A=d F /\left.d z\right|_{0}=0$.

3. Unbounded dissipative operators. We now consider unbounded linear operators satisfying the dissipative condition (1.5). Unlike the bounded case, the property of being dissipative for an unbounded operator on a Banach space $\mathfrak{X}$ will in general depend on the particular semi inner-product (compatible with the norm of $\mathfrak{X}$ ) that is used. As we have already mentioned such operators play an important role in the theory of semi-groups of operators (see [3]).

LEMmA 3.1. If $A$ is dissipative and $\lambda>0$, then $(\lambda I-A)^{-1}$ exists and is bounded with norm $\leqq \lambda^{-1}$.

Proof. Suppose $f=\lambda y-A y$. Then

$$
\lambda|y|^{2}=\lambda[y, y] \leqq \operatorname{re}[\lambda[y, y]-[A y, y]]=\operatorname{re}[f, y] \leqq|f||y| .
$$

THEOREM 3.1. A necessary and sufficient condition for a linear operator $A$ with dense domain to generate a strongly continuous semigroup of contraction operators is that $A$ be dissipative and that $\Re(I-A)=\mathfrak{X}$.

Proof. Suppose $[S(t) ; t \geqq 0]$ is a strongly continuous semi-group of contraction operators. Then

$$
r e[(S(t) x-x), x]=r e[S(t) x, x]-|x|^{2} \leqq 0
$$

so that for $x \in \mathfrak{D}(A)$

$$
\operatorname{re}[A x, x]=\lim _{t \rightarrow 0^{+}} r e t^{-1}[(S(t) x-x), x] \leqq 0 .
$$

Thus the infinitesimal generator $A$ is dissipative. Moreover it is known (see [3; Theorem 12.3.1]) that $\mathfrak{D}(A)$ is dense and that $\mathfrak{R}(I-A)=\mathfrak{X}$. Conversely if $A$ is dissipative, we see by Lemma 3.1 that $(\lambda I-A)^{-1}$ is. of norm $\leqq \lambda^{-1}$ for all $\lambda>0$. By assumption $\mathfrak{R}(I-A)=\mathfrak{X}$ so that $\lambda=1$ is in the resolvent set of $A$. Denoting the resolvent of $A$ at $\lambda$ by $R(\lambda ; A)$, it readily follows (see [3; Cor. 2 to Theorem 5.8.4)] that 


$$
R(\lambda ; A)=R(1 ; A)(I+(\lambda-1) R(1 ; A))^{-1}
$$

for $|\lambda-1|<1$. Since the dissipative property now implies $|R(\lambda ; A)| \leqq$ $\lambda^{-1}$ for $|\lambda-1|<1$, the method of analytic continuation shows that $R(\lambda ; A)$ exists for all $\lambda>0$ and is of norm $\leqq \lambda^{-1}$ for all such $\lambda$. Since $\mathfrak{D}(A)$ is assumed dense in $\mathfrak{X}$, it now follows from the Hille-Yosida theorem [3; Theorem 12.3.1] that $A$ generates a strongly continuous semigroup of contraction operators.

Corollary. If $A$ is a densely defined closed linear operator and if both $A$ and $A^{*}$ are dissipative, then $A$ generates a strongly continuous semi-group of contraction operators.

Proof. In view of the previous theorem, it suffices to show that the range of $I-A$ is all of $\mathfrak{X}$. However since $(I-A)^{-1}$ is closed (with $A$ ) and bounded, $\Re(I-A)$ will in any case be a closed linear subspace of $\mathfrak{X}$. Hence if it is not all of $\mathfrak{X}$ there will exist a non-trivial $x_{0}^{*} \in \mathfrak{X}^{*}$ such that $\left(x-A x, x_{0}^{*}\right)=0$ for all $x \in \mathfrak{D}(A)$. As a consequence $x_{0}^{*} \in \mathfrak{D}\left(A^{*}\right)$ and $x_{0}^{*}-A^{*} x_{0}^{*}=0$. The dissipativeness of $A^{*}$ then implies $x_{0}^{*}=0$ (Lemma 3.1), contrary to the choice of $x_{0}^{*}$. It follows that $\Re(I-A)=\mathfrak{X}$.

REMARK 1. Since being a generator of a semi-group of contraction operators is independent of the choice of semi inner-product, we may conclude that an operator $A$ with dense domain and $\Re(I-A)=\mathfrak{X}$ which is dissipative with respect to one semi inner-product, will be dissipative with respect to any other semi inner-product compatible with the norm of $\mathfrak{X}$.

REMARK 2. Let $[S(t)]$ be a strongly continuous semi-group of operators with infinitesimal generator $A$ and of local type $\omega(A)$. Setting

$$
\omega \equiv \sup _{t \rightarrow 0^{+}} t^{-1} \log |S(t)|,
$$

we see by [3; Theorem 7.11.1] that $\omega=\omega(A)$ whenever $\omega<\infty$. In this case $T(t) \equiv \exp [-\omega] S(t)$ defines a semi-group of contraction operators with infinitesimal generator $A^{\prime}=A-\omega I$. By the above theorem $A^{\prime}$ will be dissipative so that

$$
0 \geqq \theta\left(A^{\prime}\right)=\theta(A)-\omega .
$$

On the other hand if $A$ is a generator, then so is $A^{\prime \prime}=A-\theta(A) I$ and since $A^{\prime \prime}$ is obviously dissipative, Theorem 3.1 implies that $\exp [-\theta(A) t]$ $S(t)$ is a contraction operator. As a consequence $[S(t)]$ is of local type $\leqq \theta(A)$ and combining this with (3.2) we obtain

$$
\theta(A)=\omega(A) .
$$


By way of illustrating the above discussion we consider semi-groups of contraction operators on the space $C(S)$ of real-valued continuous functions defined on a compact Hausdorff space $S$ with infinitesimal generator $A$ taking the constant functions into 0 . We shall show that the semi-group operators are necessarily positive. It suffices to prove (see [3; Theorem 11.7.2.]) that $R(\lambda ; A)$ is positive for $\lambda>0$. For a given $f \in C(S)$ with a maximum of $|f(x)|$ at $x_{0}$ we define $W f$ by $(g, W f)=$ $g\left(x_{0}\right) f\left(x_{0}\right)=[g, f]$. Suppose for $y \in \mathcal{D}(A)$ and fixed $\lambda>0$ that $\lambda y-A y=$ $f \geqq 0$ and that $y(x)$ has a minimum at $x_{0}$. Then for $c$ sufficiently large $|y(x)-c|$ assumes its maximum value at $x_{0}$. In view of Remark 1 we can choose $W(y-c)$ so that

$$
(A(y-c), W(y-c))=[A(y-c)]\left(x_{0}\right)\left(y\left(x_{0}\right)-c\right) .
$$

Since $A$ is dissipative this expression is $\leqq 0$ and this together with $A \mathrm{c}=$ 0 implies $(A y)\left(x_{0}\right) \geqq 0$. Hence

$$
y\left(x_{0}\right) \geqq y\left(x_{0}\right)-(A y)\left(x_{0}\right) \geqq f\left(x_{0}\right) \geqq 0,
$$

which is the desired result.

It is clear from Lemma 3.1 that if $A$ is dissipative then $I-A$ is one-to-one. Consequently when $\Re(I-A)=\mathfrak{X}$ the operator $A$ is necessarily maximal dissipative in the sense of not having a proper dissipative extension. Thus it follows from Theorem 3.1 that the generators of strongly continuous semi-groups of contraction operators are maximal dissipative operators with dense domains. The converse has been shown to be true when $\mathfrak{X}$ is a Hilbert space (see [5]). Unfortunately, as the example given below shows, this converse is not in general valid.

Example. Let $\mathfrak{X}=C_{0}[0,1]$ be the continuous real-valued functions. on $[0,1]$ which vanish at $x=0$ and 1 . For each $f \in C_{0}[0,1]$ pick an $x_{0}$. at which $|f(x)|$ attains its maximum and define the semi inner-product. by $[g, f]=g\left(x_{0}\right) f\left(x_{0}\right)$. Finally we set

$$
\begin{aligned}
A y & =y^{\prime} \\
\mathfrak{D}(A) & =\left[y ; y(x) \text { continuously differentiable, } y \text { and } y^{\prime} \text { in } C_{0}[0,1]\right] .
\end{aligned}
$$

One readily verifies that $A$ is dissipative (in fact $[A y, y]=0, y \in \mathfrak{D}(A)$ and that $\mathfrak{D}(A)$ is dense in $C_{0}[0,1]$. On the other hand

$$
\Re(I-A)=\left[f ; f \in C_{0}[0,1], \int_{0}^{1} e^{-x} f(x) d x=0\right],
$$

which is obviously not even dense in $C_{0}[0,1]$. We now prove that $A$ is maximal dissipative. If this were not the case there would exist a proper dissipative extension, say $\widetilde{A}$. Suppose $u$ belongs to $\mathfrak{I}(\widetilde{A})$ but not to $\mathfrak{D}(A)$ and set $g=u-\widetilde{A} u$. For fixed $x_{0}, 0<x_{0}<1$, we construct an 
$f \in C_{0}[0,1]$ such that $f(x)=g(x)$ for $x>x_{0}$ and such that $\int_{0}^{1} e^{-x} f(x) d x=$ 0 . Then $f \in \mathfrak{R}(I-A)$ so that there is a $y \in \mathfrak{D}(A)$ with $y-A y=f$. Finally for $w=y-u$ we see that $[\tilde{A} w](x)=w(x)$ for $x>x_{0}$. Suppose now that $w(x)$ has a positive relative maximum at $x_{1}>x_{0}$. In this case we choose a smooth $h, 0 \leqq h(x) \leqq 1$, so that $x_{1}$ is a maximum of $w(x)$ on the support of $h$ (contained in $\left[x_{0}, 1\right]$ ) and so that $h(x)$ equals one only at $x_{1}$. Then $h \in \mathfrak{D}(A)$ and $[A h]\left(x_{1}\right)=0$. For sufficiently large positive $c$, it is clear that $|w(x)+c h(x)|$ has its only maximum at $x_{1}$. For such $c$,

$$
[\tilde{A}(w+c h),(w+c h)]=\left\{[\widetilde{A} w]\left(x_{1}\right)+c[A h]\left(x_{1}\right)\right\}\left\{w\left(x_{1}\right)+\operatorname{ch}\left(x_{1}\right)\right\}>0
$$

since $[\tilde{A} w]\left(x_{1}\right)=w\left(x_{1}\right)>0$ by assumption. The relation (3.4) is contrary to $\widetilde{A}$ being dissipative. Thus $w(x)$ can not have positive relative maximum (nor negative relative minimum) for $x>x_{0}$ and hence if $w(x)$ is not identically zero for $x \in\left(x_{0}, 1\right)$ there will be a portion of this interval for which it is, say, positive and decreasing. Suppose $w\left(x_{1}\right)>0, x_{1}>x_{0}$. We again choose $h$ as above (with the additional assumption that $h^{\prime \prime}(x)<0$ near $\left.x_{1}\right)$ and we see that for $c$ sufficiently large, $|w(x)+\operatorname{ch}(x)|$ will have its maximum at a point $x_{c}$ just to the left of $x_{1}$ where $h^{\prime}(x) \geqq 0$. Again we see by (3.4), with $x_{1}$ replaced by $x_{c}$, that this is impossible and we conclude that $w(x)=0$ for all $x>x_{0}$. This implies that $[\widetilde{A} u](x)=$ $[A y](x)=y^{\prime}(x)=u^{\prime}(x)$ for all $x>x_{0}$, and since $x_{0}$ was quite arbitrary we see that $\widetilde{A} u=u^{\prime}$. This shows that $u$ actually lies in $\mathscr{D}(A)$ and hence that $A$ is maximal dissipative.

Because of the important role played by the numerical range in these considerations it is of interest to obtain independent estimates for the supremum of the real part of the numerical range of $A$, that is for $\theta(A)$. The following result generalizes the relation (1.7) which has been shown by Lumer [4] to hold for all bounded operators on a Banach space.

Lemma 3.2. If $A$ is a linear operator, then

$$
\sup _{\substack{|x|=1 \\ x \in \mathcal{D}^{\left(A^{2}\right)}}} \varlimsup_{t \rightarrow 0^{+}} t^{-1}(|x+t A x|-1) \leqq \theta(A) \leqq \sup _{\substack{|x|=1 \\ x \in \mathcal{D}(A)}} \lim _{t \rightarrow 0^{+}} t^{-1}(|x+t A x|-1)
$$

Proof. It is clear for $x \in \mathfrak{D}(A)$ and $|x|=1$ that

$$
|[x+t A x, x]|=\{1+2 \operatorname{tre}[A x, x]+o(t)\}^{1 / 2}=1+\operatorname{tre}[A x, x]+o(t),
$$

and hence that

$$
r e[A x, x]=\lim _{t \rightarrow 0^{+}} t^{-1}\{|[x+t A x, x]|-1\} \leqq \lim _{t \rightarrow 0^{+}} t^{-1}(|x+t A x|-1) .
$$

This establishes the right inequality in (3.5). The left inequality is trivial if $\theta(A)=\infty$. If this is not case, we set $y=x+t A x$. Then for 
$x \in \mathfrak{D}\left(A^{2}\right)$ and $t<\{\theta(A)\}^{-1}$ we have

$$
|y||y-t A y| \geqq|[y-t A y, y]| \geqq|y|^{2}-\operatorname{tre}[A y, y] \geqq\{1-t \theta(A)\}|y|^{2} .
$$

For $|x|=1$, the above inequality together with

$$
|y-t A y|=\left|x-t^{2} A^{2} x\right|=1+o(t)
$$

gives

$$
t^{-1}(|x+t A x|-1) \leqq t^{-1}\left\{[1+o(t)][1-t \theta(A)]^{-1}-1\right\}=\theta(A)+o(1) .
$$

which is the desired result.

In case $A$ is bounded we see that (3.6) holds uniformly in $x,|x|=$ 1 , so that (3.7) now implies

$$
t^{-1}(|I+t A|-1)=\sup _{|x|=1} t^{-1}(|x+t A x|-1) \leqq \theta(A)+o(1) .
$$

It is also clear that the right inequality in (3.5) continues to hold if we interchange lim inf and sup. We therefore obtain as a

CoRollary. If $A$ is a bounded linear operator, then

$$
\theta(A)=\lim _{t \rightarrow 0^{+}} t^{-1}(|I+t A|-1) .
$$

Of primary importance in applying the theory of semi-groups is a convenient criterion for an operator to be a semi-group generator. In the case of a dissipative operator $A$ with dense domain we have seen that $\mathfrak{R}(I-A)=\mathfrak{X}$ is sufficient but that maximality is not. We now give another sufficient condition covering a fairly large class of operators.

THEOREM 3.2. Let $A$ be a closed dissipative operator and suppose that

$$
\left|A^{n} x\right|^{1 / n}=o(n)
$$

on a dense subset $\mathfrak{D}$ of $\mathfrak{D}\left(A^{\infty}\right)$. Then $A$ generates a strongly continuous semi-group of contraction operators.

Proof. We begin by defining

$$
S(t) x=\sum_{n=0}^{\infty} t^{n} / n ! A^{n} x
$$

for all $x \in \mathfrak{D}$. It is clear from the abstract Cauchy-Hadamard theorem (see [3; Theorem 3.11.4]) that $\mathfrak{D}$ is precisely the subset of $\mathfrak{D}(A)$ for which the above series converges for all $t \geqq 0$. As a consequence $S(t)$ is a linear and strongly continuous on $D$. Moreover since $A$ is assumed 
closed it is readily verified that

$$
A^{k} S(t) x=\sum_{n=0}^{\infty} t^{n} / n ! A^{k+n} x
$$

Therefore

$$
\exp (s A)[S(t) x]=\sum_{k=0}^{\infty} s^{k} / k ! \sum_{n=0}^{\infty} t^{n} / n ! A^{k+n} x
$$

and since this double sum is absolutely convergent for all $s$ it follows that $S(t) x$ belongs to $\mathfrak{D}$. Rearranging terms we verify that $S(s)[S(t) x]=$ $S(s+t) x$. Thus $S(t)$ defines a strongly continuous semi-group of operators on $\mathfrak{D}$.

We next show that $S(t)$ is a contraction operator on $\mathfrak{D}$. In fact the series form of $S(s)$ implies that

$$
|S(s) S(t) x|-|S(t) x|=|S(t) x+s A S(t) x|-|S(t) x|+O\left(s^{2}\right) .
$$

Hence by Lemma 3.2 we may conclude that

$$
\begin{aligned}
& \varlimsup_{s \rightarrow 0^{+}} s^{-1}[|S(s+t) x|-|S(t) x|] \\
& \leqq \sup [r e[A y, y] ;|y|=|S(t) x|, y \in \mathscr{D}(A)] \leqq 0 .
\end{aligned}
$$

Thus $|S(t) x|$ is a non-increasing function of $t$ and since $S(0) x=x$ we conclude that $|S(t) x| \leqq|x|$ for all $t \geqq 0 .^{3}$ Since $D$ is dense by hypothesis, we see that $S(t)$ has a unique closed linear extension on $\mathfrak{X}$ which is again a contraction operator. To simplify our notation we shall also denote this extension by $S(t)$. It is clear that the so defined operators from a strongly continuous semi-group of contraction operators on $\mathfrak{X}$.

It remains to show that the generator of this semi-group, which we denote by $B$, is actually equal to $A$. Now for $x \in \mathfrak{D}$ it is clear that

$$
\lim _{t \rightarrow 0^{+}} t^{-1}[S(t) x-x]=A x
$$

so that $B$ extends $A$ cut down to $\mathfrak{D}$. On the other hand it is well known (see [3; Theorem 10.3.3]) that for $x \in \mathfrak{D}(B)$

$$
S(t) x-x=\int_{0}^{t} S(\sigma) B x d \sigma .
$$

For $x \in \mathfrak{D}$ it is clear that $S(\sigma) A x=A S(\sigma) x$ and since $A$ is closed we have by [3; Theorem 3.3.2]

$$
\int_{0}^{t} S(\sigma) A x d \sigma=A \int_{0}^{t} S(\sigma) x d \sigma
$$

${ }^{3}$ Here we make use of the fact that a continuous real-valued function having a nonpositive upper right hand derivative at every point of an interval is non-increasing in that interval. See for instance Titchmarsh, "Theory of Functions" p. 354. 
Each $x \in \mathfrak{D}(B)$ can be approximated by a sequence $\left\{x_{n}\right\} \subset \mathfrak{D}$. Again using the closure of $A$ together with the above remarks we get

$$
S(t) x-x=A \int_{0}^{t} S(\sigma) x d \sigma, \quad x \in \mathfrak{D}(B) .
$$

Thus

$$
B x=\lim _{t \rightarrow 0^{+}} t^{-1}[S(t) x-x]=\lim _{t \rightarrow 0^{+}} A\left[t^{-1} \int_{0}^{t} S(\sigma) x d \sigma\right]
$$

and since $t^{-1} \int_{0}^{t} S(\sigma) x d \sigma \rightarrow x$ we conclude that $x \in \mathfrak{D}(A)$ and that $A x=B x$; in other words $A \supset B$. However $A$ is dissipative and $B$ is maximal dissipative and therefore $A=B$.

REMARK 1. Since the smallest closed extension of $A$ cut down to (D) satisfies the hypothesis of the theorem, it follows that $A$ is this smallest closed extension.

REMARK 2. The hypothesis of the theorem is always satisfied by generators of groups of operators. In order to verify this, let $[S(t)$; $-\infty<t<\infty$ ] be a strongly continuous group of operators with infinitesimal generator $A$ and set

$$
y_{\varepsilon}=\int_{-\infty}^{\infty} k(t, \varepsilon) S(t) x d t, \quad x \in \mathfrak{X},
$$

where

$$
k(t, \varepsilon)=(\pi \varepsilon)^{-1 / 2} \exp \left(-t^{2} / \varepsilon\right) .
$$

Obviously $y_{\varepsilon} \rightarrow x$ as $\varepsilon \rightarrow 0^{+}$so that the set $\mathfrak{D}_{0}$ of elements of the form $y_{\varepsilon}$ is dense in $\mathfrak{X}$. On the other hand by [3; Theorem 10.3.4]

$$
A^{n} y_{\varepsilon}=(-1)^{n} \int_{-\infty}^{\infty} k^{(n)}(t, \varepsilon) S(t) x d t
$$

Now it is known (see [3; p. 306]) that $|S(t)|=M \exp (\omega|t|)$ for some constants $\omega$ and $M$. Also, there is the estimate (see [3; pp. 571-572])

$$
\int_{-\infty}^{\infty} \exp \left(t^{2} / 2 \varepsilon\right)\left|k^{(n)}(t, \varepsilon)\right| d t=0\left[\left(2^{n} n !\right)^{1 / 2} n^{1 / 4}\right] \text {, }
$$

for fixed $\varepsilon$. Since $M^{\prime} \exp \left(t^{2} / 2 \varepsilon\right)$ majorizes $M \exp (\omega|t|)$ for $M^{\prime}$ sufficiently large, we see that

$$
\left|A^{n} y\right|^{1 / n}=O\left(n^{1 / 2}\right)=o(n) .
$$

The set $\mathfrak{D}$ of the theorem obviously contains $\mathfrak{D}_{0}$, so that it too is dense.

REMARK 3. The hypothesis of Theorem 4.1 is not always satisfied 
by semi-group generators. In fact let $\mathfrak{X}=L_{1}(0, \infty)$ and set

$$
(S(t) f)(x)= \begin{cases}f(x-t), & x \geqq t, \\ 0, & 0 \leqq x<t .\end{cases}
$$

Then the infinitesimal generator $A$ is defined by

$$
\begin{aligned}
\mathfrak{D}(A) & =\left[f ; f(x) \text { absolutely continuous, } f(x) \in L_{1}(0, \infty), f(0)=0\right] \\
(A f)(x) & =-f^{\prime}(x) .
\end{aligned}
$$

A function $f$ in $\mathfrak{D}\left(A^{\infty}\right)$ is therefore infinitely differentiable with $f^{(n)}(0)=$ 0 for all $n \geqq 0$. Suppose now that $f$ satisfies

$$
\left|A^{n} f\right|^{1 / n}=o(n) \text {. }
$$

Then employing the Taylor expansion with remainder we see that

$$
f(x)=(n !)^{-1} \int_{0}^{x}(x-s)^{n} f^{(n)}(s) d s .
$$

Consequently

$$
|f(x)| \leqq x^{n} / n ! \int_{0}^{\infty}\left|f^{(n)}(s)\right| d s=x^{n} / n !\left|A^{n} f\right| .
$$

For fixed $\varepsilon, x>0$ we can choose $n$ so that $\left|A^{n} f\right| \leqq(\varepsilon n / e x)^{n}$. In this case

$$
|f(x)| \leqq \varepsilon^{n}(n / e)^{n}(n !)^{-1} \leqq \varepsilon^{n},
$$

and $n$ being arbitrary, we conclude that $f(x) \equiv 0$. Thus the set $D$ of the theorem contains only the zero vector in this example.

Lemma 3.3. If $A$ is dissipative with dense domain, then $A$ has a smallest closed linear extension $\bar{A}$.

Proof. If $A$ does not have a closed extension, then there is a sequence $\left\{x_{n}\right\} \subset \mathfrak{D}(A)$ such that $x_{n} \rightarrow 0$ and $A x_{n} \rightarrow y,|y|=1$. Choose $u \in \mathfrak{D}(A)$ such that $|u-y|<1 / 2,|u|=1$. It is clear that the adjoint space $\mathfrak{X}^{*}$ will contain a weak* limit point $v_{c}^{*}$ of the bounded set of functionals $\left\{W\left(u+c x_{n}\right)\right\}$; here $W$ defines the semi inner-product on $X$. The functional $v_{c}^{*}$ has the properties

$$
\begin{aligned}
\left|v_{c}^{*}\right| & \leqq \underline{\lim }\left|W\left(u+c x_{n}\right)\right|=1, \\
\left(u, v_{c}^{*}\right) & =\lim \left(u+c x_{n}, W\left(u+c x_{n}\right)\right)=1 .
\end{aligned}
$$

Furthermore we see that

$$
\left.r e\left(A u+c y, v_{c}^{*}\right) \leqq \varlimsup \lim r e\left(A u+c x_{n}\right), W\left(u+c x_{n}\right)\right) \leqq 0 .
$$

On the other hand $\left(y, v_{c}^{*}\right)=\left(u, v_{c}^{*}\right)+\left(y-u, v_{c}^{*}\right) \geqq 1-1 / 2=1 / 2$ so that 
(3.9) can not hold if $c$ has been chosen greater than $2|A u|$.

LEMMA 3.4. If $A$ is a dissipative operator with dense domain and smallest closed extension $\bar{A}$, then there exists a semi inner-product relative to which $\bar{A}$ is dissipative.

Proof. Let $x$ belong to $\mathfrak{D}(\bar{A})$ but not to $\mathfrak{D}(A)$. Then there is a sequence $\left\{x_{n}\right\} \subset \mathcal{D}(A)$ such that $x_{n} \rightarrow x$ and $A x_{n} \rightarrow \bar{A} x$. Let $v^{*}$ be a weak* limit point of the set $\left\{W x_{n}\right\}$. Then as above

$$
\begin{gathered}
\left|v^{*}\right| \leqq \lim \left|W x_{n}\right|=|x|, \\
\left(x, v^{*}\right)=\lim \left(x_{n}, W x_{n}\right)=|x|^{2},
\end{gathered}
$$

so that $\left|v^{*}\right|=|x|$. Moreover

$$
r e\left(\bar{A} x, v^{*}\right) \leqq \varlimsup \lim r e\left(A x_{n}, W x_{n}\right) \leqq 0
$$

and hence if we redefine $W$ in the set of all such $x$ by setting $W x=$ $v^{*}$, then $\bar{A}$ will be dissipative relative to the semi inner product induced by the new $W$.

4. A convexity theorem. The Marcel Riesz convexity theorem (See [2; p. 525]) when applied to semi-groups of operators leads to a simple convexity theorem for the local types of these semi-groups and hence (by (3.3)) for the supremum of the real parts of the numerical ranges of the corresponding infinitesimal generators.

Theorem 4.1. Suppose $[S(t)]$ defines a strongly continuous semigroup of operators of finite local type $\omega_{p}$ on $L_{p}$ and of finite local type $\omega_{q}$ on $L_{q}, 1 \leqq p \leqq q \leqq \infty$. Then $[S(t)]$ defined on $L_{p} \cap L_{q}$ can be extended to be a strongly continuous semi-group of local type

$$
\omega_{s} \leqq \alpha \omega_{p}+\beta \omega_{p}, \quad s^{-1}=\alpha p^{-1}+\beta q^{-1},
$$

on $L_{s}$ for $\alpha, \beta \geqq 0, \alpha+\beta=1$.

Proof. The inequality (4.1) follows directly from (3.1) and the Riesz convexity theorem which gives

$$
|S(t)|_{s} \leqq|S(t)|_{p}^{\alpha}|S(t)|_{q}^{\beta} .
$$

Now for any $x \in L_{p} \cap L_{q}$ we see that $S(t) x$ is strongly continuous in both $L_{p}$ and $L_{q}$. A simple calculation shows that

$$
\left|S\left(t_{1}\right) x-S\left(t_{2}\right) x\right|_{s} \leqq\left|S\left(t_{1}\right) x-S\left(t_{2}\right) x\right|_{p}^{\alpha}\left|S\left(t_{1}\right) x-S\left(t_{2}\right) x\right|_{q}^{\beta}
$$

so that $S(t) x$ is strongly continuous in $L_{s}$ for $x \in L_{p} \cap L_{q}$. Since $L_{p} \cap L_{q}$ is dense in $L_{s}$ and since $|S(t)|_{s}$ is bounded by (4.2) on compact subsets 
of $[0, \infty)$, it follows that $[S(t)]$ is strongly continuous on all of $L_{s}$.

REMARK 1. If $x \in L_{p} \cap L_{q}$ and $\lambda>\max \left(\omega_{p}, \omega_{q}\right)$, then

$$
\int_{0}^{\infty} \exp (-\lambda t) S(t) x d t
$$

converges in $L_{s}$ to $R(\lambda ; A) x$ (see [3; Theorem 11.5.1] for all $s \in[p, q]$. Thus the set

$$
\mathfrak{D}_{0} \equiv R(\lambda ; A)\left[L_{p} \cap L_{q}\right]
$$

lies in $L_{s}$. Given any $x \in L_{s}$ there is a sequence $\left\{x_{n}\right\} \subset L_{p} \cap L_{q}$ which converges to $x$ in $L_{s}$. Clearly $R(\lambda ; A) x_{n} \rightarrow R(\lambda ; A) x$ in $L_{s}$ and

$$
A R(\lambda ; A) x_{n}=\lambda R(\lambda ; A) x_{n}-x_{n} \rightarrow \lambda R(\lambda ; A) x-x=A R(\lambda ; A) x
$$

also in $L_{s}$. It follows from this that the $L_{s}$ closure of the restriction of $A$ to $\mathfrak{D}_{0}$ is the generator of $[S(t)]$ in $L_{s}$ for each $s \in[p, q]$.

REMARK 2. In applications it is sometimes convenient to apply this theorem with $L_{\infty}$ replaced by $C(S)$; here $S$ is a locally compact Hausdorff space and $C(S)$ consists of all continuous real or complex valued functions defined on $S$ and vanishing at infinity. For a regular $\sigma$-finite measure $\mu$ defined on the Baire subsets $\Sigma$ of $S$, we set $L_{p}=L_{p}(S, \Sigma, \mu)$. Suppose $T$ is defined on $C(S) \cap L_{p}, 1 \leqq p<\infty$, and bounded when considered as an operator in either $C(S)$ or $L_{p}$. In this case $T$ can also be thought of as an operator in $L_{\infty}$ with its $C(S)$ bound. As such it can be extended to the simple functions without increasing its bound. (A simple function takes on only a finite set of values and is different from zero only on a set of finite measure.) In fact let $f$ be a simple function. Then $f \in L_{p}$ so that $g=T f \in L_{p}$. We shall show that $g \in L_{\infty}$ and $|g| \leqq|T|_{\sigma}|f|_{\infty}$. It is clear that one can construct a sequence $\left\{f_{n}\right\} \subset$ $C(S) \cap L_{p}$ such that $\left|f_{n}\right|_{\infty} \leqq|f|_{\infty}$ and $f_{n} \rightarrow f$ in $L_{p}$. Since $T f_{n}$ converges to $g$ in $L_{p}$ a subsequence (which we renumber) will converge almost everywhere to $g$. Now $\left|T f_{n}\right|_{\infty} \leqq|T|_{o}\left|f_{n}\right|_{\infty} \leqq|T|_{o}|f|_{\infty}$, so that $g \in L_{\infty}$ and $|g|_{\infty} \leqq|T|_{o}|f|_{\infty}$ which was to be proved. This shows that $T$ is already defined on the simple functions on $L_{\infty}$ with norm $|T|_{\sigma}$. On the other hand in the proof of the Riesz convexity theorem for the $q=\infty$ case, the $L_{s}$ extension is obtained from the $L_{\infty}$ behavior on simple functions. It therefore follows that Theorem 4.1 also holds with $L_{\infty}$ replaced by $C(S)$.

TheOREM 4.2. For $1 \leqq p \leqq q \leqq \infty$ suppose that $A_{p}$ and $A_{q}$ generate strongly continuous semi-groups $\left[S_{p}(t)\right]$ and $\left[S_{q}(t)\right]$ of finite local types $\omega_{p}$ and $\omega_{q}$, respectively. For $r=p$ or $q$ let 


$$
\begin{aligned}
\mathfrak{D}\left(A_{0}\right) & =\left[x ; x \text { and } A_{r} x \in L_{p} \cap L_{q}\right], \\
A_{0} x & =A_{r} x,
\end{aligned}
$$

and suppose that the restrictions of $A_{p}$ and $A_{q}$ to $\mathfrak{D}\left(A_{0}\right)$ are equal. Finally for all sufficiently large $\lambda$ suppose that the range of $\left(\lambda I-A_{0}\right)$ is precisely $L_{p} \cap L_{q}$. Then for $s^{-1}=\alpha p^{-1}+\beta q^{-1}, \alpha, \beta \geqq 0, \alpha+\beta=1$, the smallest closed extension of $A_{0}$ considered as an operator in $L_{s}$ exists and generates a strongly continuous semi-group of finite local type $\leqq \alpha \omega_{p}+\beta \omega_{q}$. Actually $S_{p}(t)$ and $S_{q}(t)$ are identical on $L_{p} \cap L_{q}$ and the $L_{s}$ generated semi-group is simply the smallest closed $L_{s}$ extension of the restriction of $S_{p}(t)$ or $S_{q}(t)$ to $L_{p} \cap L_{q}$.

Proof. We first show that the restrictions of $S_{p}(t)$ and $S_{q}(t)$ to $L_{p} \cap L_{q}$ are identical. To this end set $R_{0}(\lambda)=\left(\lambda I-A_{0}\right)^{-1}$. Then for sufficiently large $\lambda, R_{0}(\lambda)$ is defined on all $L_{p} \cap L_{q}$ to $\mathfrak{D}\left(A_{0}\right) \subset L_{p} \cap L_{q}$. It is also a restriction of both $R\left(\lambda ; A_{p}\right)$ and $R\left(\lambda ; A_{q}\right)$ which are in norm $\leqq\left(\lambda-\max \left(\omega_{p}, \omega_{q}\right)\right)^{-1}$. Consequently the sum

$$
S_{0}(\lambda, t) x \equiv e^{-\lambda t} \sum_{n=0}^{\infty} \frac{\left(\lambda^{2} t\right)^{n}}{n !}\left[R_{0}(\lambda)\right]^{n} x, \quad x \in L_{p} \cap L_{q},
$$

converges in both $L_{p}$ and $L_{q}$ so that $S_{0}(\lambda, t)\left[L_{p} \cap L_{q}\right] \subset L_{p} \cap L_{q}$. Passing to the limit as $\lambda \rightarrow \infty$, it is shown in the proof of the Hille-Yosida theorem (See [3; Theorem 12.3.1]) that $S_{0}(\lambda, t) x$ converges in $L_{p}$ to $S_{p}(t) x$ and in $L_{q}$ to $S_{q}(t) x$. Thus for $x \in L_{p} \cap L_{q}$

$$
S_{p}(t) x=\lim _{\lambda} S_{0}(\lambda, t) x \equiv S_{0}(t) x=S_{q}(t) x_{p} \subset L_{p} \cap L_{q} .
$$

The hypothesis of Theorem 4.1 is therefore satisfied and the $L_{s}$ extension of $S_{0}(t)$ defines a strongly continuous semi-group of operators of the required local type. Finally it is clear from the hypothesis that $R\left(\lambda ; A_{r}\right)\left[L_{p} \cap L_{q}\right]=\mathfrak{D}\left(A_{0}\right)$ for $r$ equal to both $p$ and $q$. Thus $\mathfrak{D}\left(A_{0}\right)=$ $\mathfrak{D}_{0}$ of Remark 1 above and hence the smallest closed extension of $A_{0}$ in $L_{s}$ generates the above defined semi-grup of operators.

REMARK 3. It follows from Remark 2 that Theorem 4.2 also holds with $L_{\infty}$ replaced by $C(S)$.

5. Appendix. We shall now prove a lemma on normed linear spaces, mentioned earlier, which is useful when considering different semi inner-products inducing the same norm.

Let $\mathfrak{X}$ denote any (real or complex) normed linear space, $\mathfrak{X}^{*}$ its dual. For any $p \in \mathfrak{X},|p|=1$, define

$$
C(p)=\left\{x^{*} \in \mathfrak{X}^{*} ;\left(p, x^{*}\right)=\left|x^{*}\right|=1\right\} .
$$


Lemma 5.1. Let $(\mathcal{D}$ be a dense subset of the unit sphere $S$ of $\mathfrak{X}$, and let $C_{\varepsilon}$ denote the weak * closed convex hull of $\{W x ; x \in \mathfrak{D}, \mid x-p<\varepsilon\}$; $W$ denoting as before, the mapping from $\mathfrak{X}$ to $\mathfrak{X}^{*}$ defined in $\S 1$. Then

$$
F(p) \equiv \bigcap_{\varepsilon>0} C_{\varepsilon}=C(p)
$$

Proof. First suppose that $x^{*} \in F(p)$. Then for each $\varepsilon>0$ there is a finite set $\left\{x_{i}\right\} \subset \mathcal{D},\left|x_{i}-p\right|<\varepsilon$ and numbers $\alpha_{i}, \alpha_{i} \geqq 0, \Sigma \alpha_{i}=1$ such that $\left|\left(p, x^{*}\right)-\left(p, \Sigma \alpha_{i} W x_{i}\right)\right|<\varepsilon$. Hence

$$
\begin{aligned}
\left|\left(p, x^{*}\right)-1\right| & \leqq \varepsilon+\left|\left(p, \Sigma \alpha_{i} W x_{i}\right)-\Sigma \alpha_{i}\left(x_{i}, W x_{i}\right)\right| \\
& \leqq \varepsilon+\left|\Sigma \alpha_{i}\left(p-x_{i}, W x_{i}\right)\right| \leqq 2 \varepsilon .
\end{aligned}
$$

Thus if $x^{*} \in F(p)$, then $\left(p, x^{*}\right)=1$ and since clearly $\left|x^{*}\right| \leqq 1$ it follows that $x^{*} \in C(p)$; that is $F(p) \subset C(p)$.

Let us now show the converse. $F(p)$ is clearly convex and compact. One can without loss of generality assume that $\mathfrak{X}$ is real. In fact, if $\mathfrak{X}$ is a complex normed linear space, let $\mathfrak{X}_{r}$ denote this space regarded as real; it is then easily shown that the corresponding $C_{r}(p)$ is the set $\left\{r e x^{*} ; x^{*} \in C(p)\right\}$ and $C_{r \varepsilon}=\left\{r e x^{*}: x^{*} \in C_{\varepsilon}\right\}$. Thus, suppose $\mathfrak{X}$ is real and that $x_{0}^{*} \in C(p) \ominus F(p)$. Then by an elementary separation theorem for conpact convex sets there exists $y \in \mathfrak{X}$ such that $\left(y, x_{0}^{*}\right)>1,\left(y, x^{*}\right) \leqq 1$ for all $x^{*} \in F(p)$. Since $\mathfrak{D}$ is dense, there is a $y_{0} \in \mathfrak{D}$ such that $\left(y_{0}, x_{0}^{*}\right)>k \geqq\left(y_{0}, x^{*}\right)$ for all $x^{*}$ in $F(p)$.

Next we consider the subspace $\bar{X}$ spanned by $p$ and $y_{0}$. Since $\left(p, x_{0}^{*}\right)=$ $\left(p, x^{*}\right)=1$ for all $x^{*} \in F(p), y_{0}$ and $p$ are not colinear, and $\bar{X}$ is actually two dimensional.

If $x$ is any point on the unit surface $\bar{S}$ of $\bar{X},\left\{x_{n}\right\} \subset \mathcal{D}$ may be so chosen that $\overline{W x_{n}}$, the restriction of $W x_{n}$ to $\bar{X}$, converges so-to-speak to a support line at $x$. Hence by considering points on either side of $p$ on $\bar{S}$, it is clear that the two extreme support lines at $p$ belong to the restriction of $F(p)$ to $\bar{X}$, and since $F(p)$ is convex the support line corresponding to $\bar{x}_{0}^{*}$ belongs to $F(p)$. But this means that $\sup \left[\left(y_{0}, x^{*}\right)\right.$; $\left.x^{*} \in F(p)=\left(y_{0}, x_{0}^{*}\right)\right]$ which is contrary to one choice of $y_{0}$.

CoRollary. Let $T$ denote a bounded operator on any (real or complex) normed linear space $\mathfrak{X}$. Consider two mappins $W_{1}$ and $W_{2}$, from $\mathfrak{X}$ to $\mathfrak{X}^{*}$ defined as usual. Then the corresponding numerical ranges of $T, W_{1}(T)$ and $W_{2}(T)$ have the same closed convex hull.

Proof. In fact write $C(T)=$ closed convex hull of $\left\{\left(T x, x^{*}\right)\right.$; $\left.x^{*} \in C(x),|x|=1\right\}$; we shall show that $C(T)$ is the closed convex hull of $W_{1}(T)$ (as well as of $W_{2}(T)$ ). Clearly $W_{1}(T) \subset C(T)$. On the other hand, given $x^{*} \in C(x),|x|=1$, then by the previous lemma, we may 
choose $\left\{x_{i}^{n}\right\} \subset S, x_{i}^{n} \rightarrow x$ (uniformly in $i$ ), and $\Sigma_{i} \alpha_{i}^{n} W_{1} x_{i}^{n} \rightarrow x^{*}$, with $\alpha_{i}^{n} \geqq 0$, $\Sigma_{i} \alpha_{i}^{n}=1$. Now $\left|\Sigma_{i} \alpha_{i}^{n}\left(T x, W_{1} x_{i}^{n}\right)-\Sigma_{i} \alpha_{i}^{n}\left(T x_{i}^{n}, W_{1} x_{i}^{n}\right)\right| \leqq|T|_{i} \sup \left|x-x_{i}^{n}\right| \rightarrow$ 0 and $\Sigma \alpha_{i}^{n}\left(T x, W_{1} x_{i}^{n}\right) \rightarrow\left(T x, x^{*}\right)$ which thus belongs to the closed convex hull of $W_{1}(T)$.

\section{REFERENCES}

1. H.F. Bohnenblust and S. Karlin, Geometrical properties of the unit sphere of Banach algebras, Annals of Math., 62 (1955), 217-229.

2. N. Dunford and J. Schwartz, Linear operators, I. Interscience, 1958.

3. E. Hille and R. S. Phillips, Functional analysis and semi-groups, Amer. Math. Soc. Colloquium Publ., 31, (1957).

4. G. Lumer, Semi inner-product spaces, forthcoming.

5. R. S. Phillips, Dissipative operators and hyperbolic systems of partial differential equations, Trans. Amer. Math. Soc., 90 (1959), 193-254.

UNiversity of CALIFornia, Los ANGeles 


\title{
PACIFIC JOURNAL OF MATHEMATICS
}

\author{
EDITORS
}

\author{
Ralph S. Phillips \\ Stanford University \\ Stanford, California \\ F. H. Brownell \\ University of Washington \\ Seattle 5, Washington
}

\author{
A. L. Whiteman \\ University of Southern California \\ Los Angeles 7, California \\ L. J. PAige \\ University of California \\ Los Angeles 24, California
}

ASSOCIATE EDITORS
E. F. BECKENBACH
D. DERRY
H. L. ROYDEN
E. G. STRAUS
T. M. CHERRY
M. OHTSUKA
E. SPANIER
F. WOLF

\section{SUPPORTING INSTITUTIONS}

\author{
UNIVERSITY OF BRITISH COLUMBIA \\ CALIFORNIA INSTITUTE OF TECHNOLOGY \\ UNIVERSITY OF CALIFORNIA \\ MONTANA STATE UNIVERSITY \\ UNIVERSITY OF NEVADA \\ NEW MEXICO STATE UNIVERSITY \\ OREGON STATE COLLEGE \\ UNIVERSITY OF OREGON \\ OSAKA UNIVERSITY \\ UNIVERSITY OF SOUTHERN CALIFORNIA
}

\author{
STANFORD UNIVERSITY \\ UNIVERSITY OF TOKYO \\ UNIVERSITY OF UTAH \\ WASHINGTON STATE COLLEGE \\ UNIVERSITY OF WASHINGTON \\ * * * * \\ AMERICAN MATHEMATICAL SOCIETY \\ CALIFORNIA RESEARCH CORPORATION \\ HUGHES AIRCRAFT COMPANY \\ SPACE TECHNOLOGY LABORATORIES \\ NAVAL ORDNANCE TEST STATION
}

Mathematical papers intended for publication in the Pacific Journal of Mathematics should be typewritten (double spaced), and the author should keep a complete copy. Manuscripts may be sent to any one of the four editors. All other communications to the editors should be addressed to the managing editor, L. J. Paige at the University of California, Los Angeles 24, California.

50 reprints per author of each article are furnished free of charge; additional copies may be obtained at cost in multiples of 50 .

The Pacific Journal of Mathematics is published quarterly, in March, June, September, and December. The price per volume (4 numbers) is $\$ 12.00$; single issues, $\$ 3.50$. Back numbers are available. Special price to individual faculty members of supporting institutions and to individual members of the American Mathematical Society: $\$ 4.00$ per volume; single issues, $\$ 1.25$.

Subscriptions, orders for back numbers, and changes of address should be sent to Pacific Journal of Mathematics, 103 Highland Boulevard, Berkeley 8, California.

Printed at Kokusai Bunken Insatsusha (International Academic Printing Co., Ltd.), No. 6, 2-chome, Fujimi-cho, Chiyoda-ku, Tokyo, Japan.

\section{PUBLISHED BY PACIFIC JOURNAL OF MATHEMATICS, A NON-PROFIT CORPORATION}

The Supporting Institutions listed above contribute to the cost of publication of this Journal, but they are not owners or publishers and have no responsibility for its content or policies. 


\section{Pacific Journal of Mathematics}

\section{Vol. 11, No. 2 December, 1961}

Tsuyoshi Andô, Convergent sequences of finitely additive measures........

Richard Arens, The analytic-functional calculus in commutative topological algebras..........................................

Michel L. Balinski, On the graph structure of convex polyhedra in

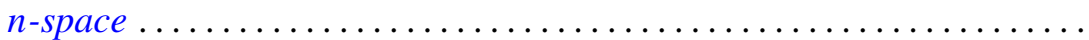

R. H. Bing, Tame Cantor sets in $E^{3}$...

Cecil Edmund Burgess, Collections and sequences of continua in the plane.

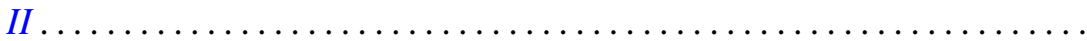

J. H. Case, Another 1-dimensional homogeneous continuum which contains an $\operatorname{arc}$

Lester Eli Dubins, On plane curves with curvature ................. 471

A. M. Duguid, Feasible flows and possible connections .............. 483

Lincoln Kearney Durst, Exceptional real Lucas sequences ................ 489

Gertrude I. Heller, On certain non-linear opeartors and partial differential equations........................................

Calvin Virgil Holmes, Automorphisms of monomial groups

Wu-Chung Hsiang and Wu-Yi Hsiang, Those abelian groups characterized by their completely decomposable subgroups of finite rank ..........

Bert Hubbard, Bounds for eigenvalues of the free and fixed membrane by finite difference methods .........................

D. H. Hyers, Transformations with bounded mth differences. .

Richard Eugene Isaac, Some generalizations of Doeblin's decomposition

John Rolfe Isbell, Uniform neighborhood retracts ..........

Jack Carl Kiefer, On large deviations of the empiric D. F. of vector chance variables and a law of the iterated logarithm...

Marvin Isadore Knopp, Construction of a class of modular functions and forms. II. . .

Gunter Lumer and R. S. Phillips, Dissipative operators in a Banach space....

Nathaniel F. G. Martin, Lebesgue density as a set function ...

Shu-Teh Chen Moy, Generalizations of Shannon-McMillan theorem ...

Lucien W. Neustadt, The moment problem and weak convergence in $L^{2}$

Kenneth Allen Ross, The structure of certain measure algebras...

James F. Smith and P. P. Saworotnow, On some classes of scalar-product algebras.

Dale E. Varberg, On equivalence of Gaussian measures. . 